•综述・

\title{
中国植物受威胁等级评估系统概述
}

\author{
单章建 $1,2 \#$ 赵莉娜 $1,2 \#$ 杨宇昌 1,2 谢 丹 ${ }^{1,2}$ 覃海宁 ${ }^{1,2 *}$ \\ 1 (中国科学院植物研究所系统与进化植物学国家重点实验室, 北京 100093) \\ 2 (中国科学院大学生命科学学院, 北京 100049)
}

\begin{abstract}
摘要: 濒危物种保护是生物多样性保护工作的重要组成部分, 而物种受威胁等级评估则是濒危物种保护的方向指 引。经过多年的发展, 物种受威胁等级的评估由定性评估逐渐向定量评估为主、定性评估为辅的方向发展。本文 综述了国内植物受威胁等级定量评估系统的研究进展, 同时介绍了国外较为成熟的IUCN红色名录评估系统、 CITES评估系统、美国自然保育协会评估系统, 提出未来制定受威胁物种定量评估标准时要兼顾以下方面: (1)等级 设置定义要明确、统一且合理; (2)评估标准应该定量化、客观且不冗余; (3)评估系统应该适应不同地理范围, 最好 能同时表达出各范围的受威胁等级; (4)评估指标要包含物种动态信息, 能定量分析物种在过去或者未来的变化。 此外, 本文认为国内的物种受威胁等级定量评估系统应该形成规范化的大纲, 加大宣传力度, 尽量将理论研究与 具体的保护行动结合起来; 同时, 我国还应该采用全球广泛应用的受威胁等级评估系统获取物种受威胁等级, 将 国内生物多样性保护工作纳入到全球范围中去。
\end{abstract}

关键词: IUCN红色名录; 濒危系数; 二级模糊评价法; 层次分析法; 专家系统; 定量评估

\section{An overview on assessment systems for threatened plants in China}

\author{
Zhangjian Shan ${ }^{1,2 \#}$, Lina Zhao ${ }^{1,2 \#}$, Yuchang Yang ${ }^{1,2}$, Dan Xie ${ }^{1,2}$, Haining Qin ${ }^{1,2 *}$ \\ 1 State Key Laboratory of Systematic and Evolutionary Botany, Institute of Botany, Chinese Academy of Sciences, Beijing \\ 100093
}

2 College of Life Science, University of Chinese Academy of Sciences, Beijing 100049

\begin{abstract}
Endangered species conservation is an important part of biodiversity conservation, heavily helped by threatened species assessment guides. The assessment of threatened level has gradually evolved from being qualitative to quantitative with qualitative supplement. This paper reviews the research progress of the domestic threatened plant species quantitative assessment system, and introduces the mature IUCN Red List Assessment System, CITES Assessment System, and the National Natural Conservation Association Assessment System. Through this, we propose future development requirements of quantitative assessment criteria for threatened species should have the following characteristics: (1) a clear, unified, and reasonable definition of category setting, (2) quantitative, objective, and non-redundant criteria for endangered categories, (3) adaptation to different geographical range while simultaneously expressing threatened category over different ranges, (4) evaluation indicators contain the dynamic information of the species which can quantitatively analyze the change of the species through time. In addition, the domestic quantitative assessment system for threatened species should form a standardized outline with increased publicity to combine theoretical research with specific conservation actions. Lastly, China should adopt the widely-used endangered rating systems to assess species threatened level and integrate domestic biodiversity conservation work into the global scale.
\end{abstract}

Key words: IUCN Red List; endangerment coefficient; fuzzy two-stage comprehensive evaluation; analytic hierarchy process; expert system; qualitative assessment

收稿日期: 2019-08-01; 接受日期: 2019-12-16

基金项目: 环境保护部生物多样性保护专项——国高等植物红色名录、国家自然科学基金(31900191)和科技部国家标本资源共享平台植物子平台 (2005DKA21401)

\# 共同第一作者 Co-first authors

* 通讯作者 Author for correspondence. E-mail: hainingqin@ibcas.ac.cn 


\section{背景}

社会经济发展中，人们对自然资源的不合理利 用与开发, 导致自然环境受到了极大的破坏。物种 生境退化, 遗传多样性丧失, 部分物种甚至面临绝 灭的风险, 因此保护生物多样性刻不容缓(Miller et al, 2006)。然而资源有限, 现阶段无法做到对所有物 种实施保护措施。因此, 以最少的资源保护尽可能 多的物种, 以最优的资源保护最需要保护的物种是 目前生物多样性保护工作的重点(蒋志刚和樊恩源, 2003)。

对物种受威胁现状进行评估并确定等级, 是确 定物种保护优先级最基本的工作。对物种进行受威 胁等级划分既能对物种的受威胁现状和生存前景 给予客观的评估, 又能简单明了地显示物种的受威 胁状态，提供开展物种保护及制定保护优先方案的 依据。长期以来, 使用较多的是定性评估系统, 但 这类评估系统由于过于主观而使用越来越少 (IUCN/UNEP et al, 1987), 取而代之的是更为客观 的定量指标(Mace \& Lande, 1991)。20世纪80年代以 来, 各国学者开始致力于物种受威胁等级定量评估 方法的研究。英国学者Perring和Farrell因提出以“威 胁” 数值大小来确定植物受威胁程度的评定方法, 成为最早的以定量指标参与评估的专家(Perring \& Farrell, 1983)。该方法提出了濒危系数(endangerment coefficient)的概念, 并采用了一系列的有效数 据评估物种的优先保护顺序。该方法在采用受威胁 数值作为评估依据时, 也允许一定程度的主观意见, 属半定量的方法。即便如此, 该方法仍开创了定量 信息参与评估的先河, 从那以后, 学者们逐渐加大 定量化的比重, 力图消除主观成分, 进一步完善并 逐渐形成能够在多尺度多类群层面上使用的定量 评估系统(IUCN/UNEP et al, 1987)。

到目前为止, 许多保护组织建立的各种全球评 估系统得到了越来越多的认可, 在许多国家得到推 广和应用(Mace \& Lande, 1991; IUCN, 2001; de Grammont \& Cuarón, 2006; Miller et al, 2007)。目前, 全球层面上使用较广泛的物种受威胁等级评估系 统主要有: (1) IUCN提出的物种红色名录评估系统。 该系统以定量化分析为主, 提出明晰客观的受威胁 等级及评估标准。de Grammont和Cuarón (2006)在对 北美各政府和组织使用最广泛的 25 个评估系统进
行评定后得出IUCN红色名录评估系统是最适合物 种受威胁等级评估的系统。Miller等(2007)对180个 《生物多样性公约》 (Convention on Biological Diversity, CBD)签约国的调查显示, 70\%的国家发 布了受威胁物种名录，其中 $78 \%$ 的评估方法采用 了IUCN红色名录评估系统的某个版本。(2)《濒危 野生动植物物种国际贸易公约》(Convention on International Trade in Endangered Species of Wild Fauna and Flora, CITES)评估系统。该系统以物种种 群大小、种群数量变化、种群面积变化等相关信息 为基础，结合物种贸易信息，将其列入三个绝灭风 险不同的等级。该系统的建立是为了控制野生动植 物的非法国际贸易(Wijnstekers, 2000)，从而达到保 护野生珍稀濒危物种的目的。(3)美国自然保育协会 (The Nature Conservancy, TNC)评估系统。该系统由 美国自然保育协会、自然遗产计划网络(Network of Natural Heritage Programs)、保育数据中心(Conservation Data Centers)等共同制定，目前在美国、加拿 大、拉丁美洲国家、加勒比海国家广泛应用。该系 统以物种稀有度、种群状况、物种受威胁因素等作 为分级因子, 将物种分为 5 个不同等级。此外，该评 估系统为适应不同地理范围, 采用了综合等级显示 方式，利用字母 G (Globally)、N (National)、S (Subnational)表示不同地理范围大小, 数字1、2、3、 4、5表示受威胁的程度依次递减。如G5S3表示全球 级别 $(G)$ 和亚国家(如省或州)级别 $(S)$ 的等级, 其中 全球级别是安全，亚国家级别是易危; 而N3S2则表 示国家级别 $(\mathrm{N})$ 和亚国家级别(S)的等级, 其中, 国 家级别是易危(3), 而亚国家级别是处于危险中(2) (Faber-Langendoen et al, 2012)。这几个广泛使用的 评估系统其评价标准不同，但在方法上有许多可以 相互借鉴之处。

我国学者使用较多的是IUCN红色名录评估系 统，该系统在我国已有30多年的应用历史。本文详 细介绍了 IUCN红色名录评估系统在我国物种受威 胁等级评估工作中的应用以及我国多类本土定量 评估系统的发展历程, 并通过分析各评估方法的优 劣, 提出可能的发展方向和建议。

IUCN红色名录评估系统及在中国的应用

IUCN 物种红色名录濒危等级和标准 (IUCN Red List Categories and Criteria, Version 3.1)将物种 
的受威胁状况分为 9 级: 绝灭(Extinct, EX)、野外绝 灭(Extinct in the Wild, EW)、极危(Critically Endangered, CR)、濒色 (Endangered, EN)、易危(Vulnerable, VU)、近危(Near Threatened, NT)、无危(Least Concern, LC)、数据缺乏(Data Deficient, DD) 和未予评估 (Not Evaluated, NE)。其中极危、濒危、易危3个受 威胁等级含有数量化的评估标准。该评估标准有 5 个量化指标, 即标准 $\mathrm{A}$ (分布范围内种群数量减少)、 标准B (地理分布范围及面积、破碎化、面积下降及 波动等)、标准 $\mathrm{C}$ (种群内成熟个体数下降或减少)、 标准D (极小种群或分布十分受限)和标准E (绝灭风 险的定量化分析) (IUCN, 2001, 2010)。

我国植物学家早在 20 世纪 80 年代就引入了 IUCN红色名录评估系统。1984年，我国学者采用 IUCN红色名录的早期系统 The IUCN Plant Red Data Book (Lucas \& Synge, 1978), 引入了濒危、稀 有和渐危 3 个等级概念, 评估了部分物种受威胁等 级, 即第一批《中国珍稀濒危保护植物名录》(国家 环境保护局和中国科学院植物研究所, 1987), 并在 1991年基于该名录扩展出版了《中国植物红皮书(第 一册): 稀有濒危植物》(傅立国, 1991)。该名录和书 籍的出版拉开了我国政府及相关组织将红色名录 系统应用于物种受威胁等级评估的序幕, 奠定了我 国受威胁植物研究和保护的基础。2001年, IUCN红 色名录评估系统新版本(3.1版)发布, 我国学者在该 版本基础上按照我国国情略作调整, 开始评估我国 各生物类群。2004年, 汪松和解炎首次使用3.1版本 的红色名录系统对我国野生动植物进行评估, 并出 版了《中国物种红色名录, 第一卷: 红色名录》(汪 松和解炎, 2004)。之后我国其他学者在工作中根据 具体情况适当调整等级设置或增加相关指标进行 综合评估。如国家林业局在2006年发布的中国野生 植物受威胁等级划分标准中, 采用了与红色名录标 准相同的等级, 仅对部分划分指标做了细微修改, 如在评估濒危等级时, 将成熟个体数下降指标由 20 年或5个世代内下降 $\geq 20 \%$ 调整为 $\geq 50 \%$ (国家林业 局, 2006)。

上述各类中国植物红色名录的出版对我国的 植物多样性保护及研究起到了重要作用, 但也存在 明显不足, 例如评估物种数量较少, 所用资料陈旧 或未能充分吸收专家参与等。为此, 2008年原环境 保护部联合中国科学院启动了《中国高等植物红色
名录》的全面评估工作。该工作是我国学者使用最 新的IUCN红色名录评估系统V3.1版首次评估中国 所有35,000余种高等植物的受威胁状况(覃海宁和 赵莉娜, 2014; Zhao et al, 2016), 共计90家科研院所 和大专院校的约 300 位植物学专家及研究生参与信 息调查或评估结果审核工作(藏春金等, 2016; 覃海 宁和赵莉娜, 2017)。除了在国家层面大尺度范围外, IUCN红色名录评估系统也越来越多地应用到地区 范围和特定类群的物种评估(Peng et al, 2008; 钟华 和周涁, 2010; 田贵全等, 2012; 黄卫昌等, 2015)。

红色名录评估系统的适用性很强，在受威胁等 级评估中一个分类单元只需要符合这些标准中的 任意一条，即可被列入相应的受威胁等级。但它也 存在相应的缺点：首先，五条标准没有全面涵盖物 种的所有信息，有些物种的信息虽然比较多，却并 不符合五个标准中的任何一个, 因此无法评出确切 的等级。其次，根据红色名录评估标准，该系统应 适用于任何类群。但事实上, 不同的类群千差万别, 从草本植物到大型哺乳动物使用的都是一套标准, 不同学者在对不同类群进行评估时，对标准中同一 个参数的理解不尽相同 (Rivers，2010; Onana \& Cheek, 2011), 这就造成了评估结果的差异。

\section{国内的植物受威胁等级定量评估系统}

除了不断引进并推广全球评估系统，我国学者 也着力于建立具有地区特色的植物受威胁等级定 量评估系统。20世纪80年代，我国便开始了植物受 威胁等级定量评估系统的研究工作。目前较为成熟 的系统有：基于濒危系数的定量评估系统(包括濒 危系数结合特别考虑种的半定量评估法和濒危系 数结合急切保护值的定量法)、层次分析法(analytic hierarchy process, AHP)、二级模糊评价法(fuzzy two-stage comprehensive evaluation)、专家系统(expert system)等。基于濒危系数的评估系统在我国使 用范围最为广泛，接受度也最高，本文将重点介绍， 同时也对其他几种定量评估系统做简要介绍。

\section{1 基于濒危系数的半定量法和定量法}

此方法对多个与受威胁信息相关的物种信息 按照一定的方式进行分级赋值，并在此基础上结合 权重获得与物种受威胁信息相关的值(系数), 即濒 危系数。这个值在获取物种保护优先顺序时承担一 定的作用。基于濒危系数的评估系统获得的物种受 
威胁等级常在物种保护优先顺序评定中使用, 按照 主观参与的程度大致分为半定量法和定量法, 前者 是以许再富和陶国达(1987)为代表的基于濒危系数 和特别考虑种的半定量法; 后者是以薛达元等(1991) 为代表的基于濒危系数和急切保护值的定量法。两 种模式在获取物种受威胁等级(濒危系数)时的原理 大致相同，但在选择物种的指标时有所不同。

\subsection{1 濒危系数 + 特别考虑种半定量法}

早在30多年前, 许再富和陶国达(1987)在研究 国内植物受威胁保护评价方法时，从植物个体生 态、种群动态、群落生态等 3 个方面, 提出以受威胁 系数结合特别考虑种的方法获得物种受威胁等级。 该方法选择植物分布区类型、研究区域内分布状 况、生物群落确限度、植物群落中的重要值和植物 群落结构等五个方面表达植物稀有、受威胁信息, 并按照信息的强弱用 5 到 1 的数量尺度进行定量标 记, 然后采取濒危系数 $\left(C_{t}\right)$ 来计算评估结果:

$$
C_{t}=\sum_{i=1}^{n} x i / \sum_{i=1}^{n} \max i
$$

其中, $x i$ 为第 $i$ 个参数的定量标记值; $\max i$ 为第 $i$ 个参 数所规定的最大值。在确定物种濒危状态时, 按以 下等级进行划分：当 $0.88 \leq C_{t}<1.00$ 时为濒危种; 当 $0.72 \leq C_{t}<0.88$ 时为渐危种; 当 $0.55 \leq C_{t}<0.72$ 时为 稀有种; $C_{t}<0.5$ 时为安全种类(许再富和陶国达, 1987)。

在获取保护级别时，该方法还同时根据《世界 自然资源保护大纲》(World Conservation Strategy, IUCN et al, 1980)中保护遗传多样性的原则, 特别考 虑较高级分类单位，如科和属的保护(Reveal, 1981)。 根据该原则, 科、属、种 3 个分类水平的优先保护性 逐渐降低，同时考虑并沿用美国佛罗里达州动植物 稀有及濒危委员会所采取的特别考虑种来校正优 先次序, 即一个物种通过濒危系数的计算已接近规 定的受威胁等级, 由于它具有重要的生态作用、科 学价值或经济价值, 故给予特别的考虑而视其为濒 危种或稀有种(崔国发等, 2000, 2007)。该方法在我 国首次以濒危系数为基础并特别考虑物种分类地 位。之后该方法在我国受威胁植物评估中有一定程 度的使用(庄平和吴荭, 1992; 李先琨, 1997; 崔国发 等, 2000; 蒋志刚和樊恩源, 2003), 但因通过特别考 虑种调整物种最终的保护级别时存在较大的主观
性，而且物种的重大价值并不能精确的界定，以致 近十多年来在我国的应用逐渐减少。

\subsection{2 濒危系数 + 急切保护值定量法}

薛达元等(1991)在对苏浙皖地区珍稀植物分级 指标的研究中提出在濒危系数基础上以急切保护 值判断物种保护优先级别。这类系统的主要思路是: 根据物种评估指标获取物种的濒危系数, 按照一定 的权重分配给包括濒危系数在内的多个系数(遗传 损失系数、利用价值系数、保护现状系数), 最终得 到物种急切保护值(也即优先保护值), 进而得到相 应的物种保护级别。濒危系数是根据国内分布频 度、研究区域内分布频度、研究区域内现存多度、 种群消失速率、种群结构等五个参考指标获取的物 种受威胁程度。该方法获取物种濒危系数时与前一 种方法相似(公式1), 只是在定量指标的选择上不 同。两者的区别主要在于获取物种保护优先级别时， 该系统提出了包括濒危系数在内的多个物种信息 相关的系数, 并按照一定的重要性分配权重后, 通 过计算公式2获得急切保护值 $(V)$ 及相应的保护系数, 判断物种保护状况, 其中濒危系数仅仅是其中一个 方面。

$$
V=0.45 C_{t}+0.25 C_{h}+0.15 C_{v}+0.15 C_{p}
$$

其中, $C_{t} 、 C_{h} 、 C_{v} 、 C_{p}$ 分别为濒危系数、遗传损失系 数、利用价值系数、保护现状系数。根据 $V$ 值的大 小, 按以下标准划分急切保护级别: $V \geq 0.7$, 一级急 切保护; $0.6 \leq V<0.7$, 二级急切保护; $0.5 \leq V<0.6$, 三级急切保护; $V<0.5$, 缓急保护(薛达元等, 1991)。

该方法将物种相关的信息进行分类，每一类单 独计算其系数, 并赋予一定的权重, 然后通过计算 公式获取物种的保护顺序值。该方法需要的物种信 息类别清晰、种类多，因而许多物种，特别是信息 较少的物种, 无法及时得出一个合理的等级。此外, 在权重分配过程中, 不同的专家有不同的侧重, 在 一定程度上也存在主观性。尽管如此，该方法在我 国使用十分广泛, 得到很多专家学者的认可(姚振 生等, 1997; 安慧君等, 2005; 方元平等, 2007; 石道 良等, 2011)。

\subsection{3 指标的选择}

不管是基于濒危系数的半定量法还是定量法, 都离不开定量指标的篮选及确定。学者在获取濒危 系数时一般采取以下几种方法选取定量指标: (1)理 论分析法: 对受威胁野生植物现状进行分析、比较, 
设计出受威胁野生植物的评估指标体系。(2)频度分 析法: 即查阅相关文献, 选择出使用频度高、具有 典型性和针对性, 而且数据可获得的指标, 设计出 受威胁野生植物的评估指标体系。(3) Delphi法、专 家咨询法和调查研究分析法: 这三种方法主要是广 泛征求专家的意见, 综合专家意见对评估指标(或 评估对象)做出一定的定量与定性的阈值设定(国政 和藏润国, 2013)。表1列出了近年来基于濒危系数评 估物种受威胁状态及保护优先顺序时所选取的评 估指标。从表中可发现, 有几个指标使用频率比较 高, 如林区内分布状况(使用率高达 $80 \%$, 下同)、国 内分布频度 $(80 \%)$ 、植物群落中的重要值(60\%)。指 标的选择多为4-6个不等(表1), 但也存在比较极端 的情况, 有些专家仅选择2个指标(何平等, 2003)。

\subsection{4 优点及不足}

总体来说, 基于濒危系数的物种受威胁等级定 量评估模式在一定程度上避免了评估结果的主观 性，评估过程简单且可重复。这种方法初步反映了 各指标的综合影响，对我国物种受威胁评估系统的 发展具重要作用, 是我国定量评估方法发展的主要 方向，但仍存在以下不足:

(1)受威胁等级与优先保护等级混淆不清。在评 估受威胁等级时, 将凸显物种重要性的信息, 如物 种演化地位、药用价值、生态价值等作为评估指标, 这种混合的指标得出的是保护优先级, 但国内很多 学者常将它作为受威胁等级使用(许再富和陶国达, 1987; 崔国发等, 2000)。物种的受威胁等级是对当 前环境下绝灭风险的评估, 而优先保护等级涉及许 多与保护行动相关的其他因素(IUCN, 2001)。

(2)受威胁指标的选择存在主观性。指标的选择 多因评价目的、对象、范围等方面的不同而不同，从 而形成不同的评估系统, 难以获得广泛的认可, 影 响系统的推广应用。

(3)等级设置及阈值划分的主观性较强。系统不 同, 受威胁等级的设置也不相同, 许再富和陶国达 (1987)将物种等级设置成安全种、稀有种、渐危种、 濒危种四类; 而崔国发等(2007)将其设定为安全 种、可能消失种、容易消失种和极易消失种, 等等。 因此，同一物种根据不同评估系统常被分为不同受 威胁等级(李景文和李俊清, 2006)。这种不统一在今 后的对比研究中会带来不便, 影响对比研究。

\section{2 层次分析法}

层次分析法是一种简单、灵活的多维准则决策 的数学方法。应用层次分析法分析问题时, 首先要 依据各要素之间的相互关系将问题分解，构建出系 统的多级递阶层次模型, 把复杂的问题系统化、层 次化。通常在使用过程中, 该法可以实现由定性到 定量的转化，为了使决策判断定量化，通常采用判 断矩阵来实现。判断矩阵表示层次结构模型中, 针 对上一层次某元素, 本层次有关元素之间两两比较 而得出的相对权重, 即哪一个更重要、重要多少等, 可以按照1-9标度法对重要性程度赋值。

应用于物种受威胁状态评估的层次分析法一 般来说包括以下过程: 第一步，确定包括总体层、 系统层、标准层及指标层四个层次的指标体系。第 二步，通过专家对这四个层面的指标建立一、二、 三级评价单元，并按照顺序求出每一评价单元中的 各指标的权重。为了尽可能减少人为因素影响, 专 家会先对每一待定指标进行初选，并分为很重要、 重要、一般和不重要等多个等级, 然后对选中的指 标直接给出权重。第三步，由专家按照递阶层次结 构对每一个上级指标所辖的下级指标两两比较其 重要程度, 得出判断矩阵。第四步, 计算评估指标 的权重及其系数, 并对其进行归一化。第五步, 求 出最大特征根和特征向量, 并进行一致性检验。最 后，计算实际指标值与标准值之间的接近程度，并 通过公式3线性加权平均合成评价综合指数 $(C)$ (国 政和藏润国, 2013)。

$$
C=\sum_{i=1}^{m}\left[\sum_{j=1}^{n}\left(\sum_{k=1}^{l}\left(F_{K} \times P_{k}\right) \times R_{j}\right) \times W_{i}\right]
$$

式中, $m 、 n 、 l$ 分别为系统层、标准层、指标层的个 数; $P_{k} 、 R_{j} 、 W_{i}$ 分别为指标层、标准层和系统层各指 标的权重; $F_{k}$ 指实际指标值与标准值之间的接近 程度。

运用层次分析法进行评价的整个过程简单明 确, 容易掌握, 因此在我国濒危植物及其他类群的 评估中得到了较多应用(陶玲等, 2001; 国政和蔵润 国, 2013; 秦立武等, 2015; 王建瑞等, 2015)。但在具 体使用时对指标的要求较高、需要物种信息量大, 不利于信息缺乏物种的受威胁状态评估, 存在理论 与实践脱节的情况。 


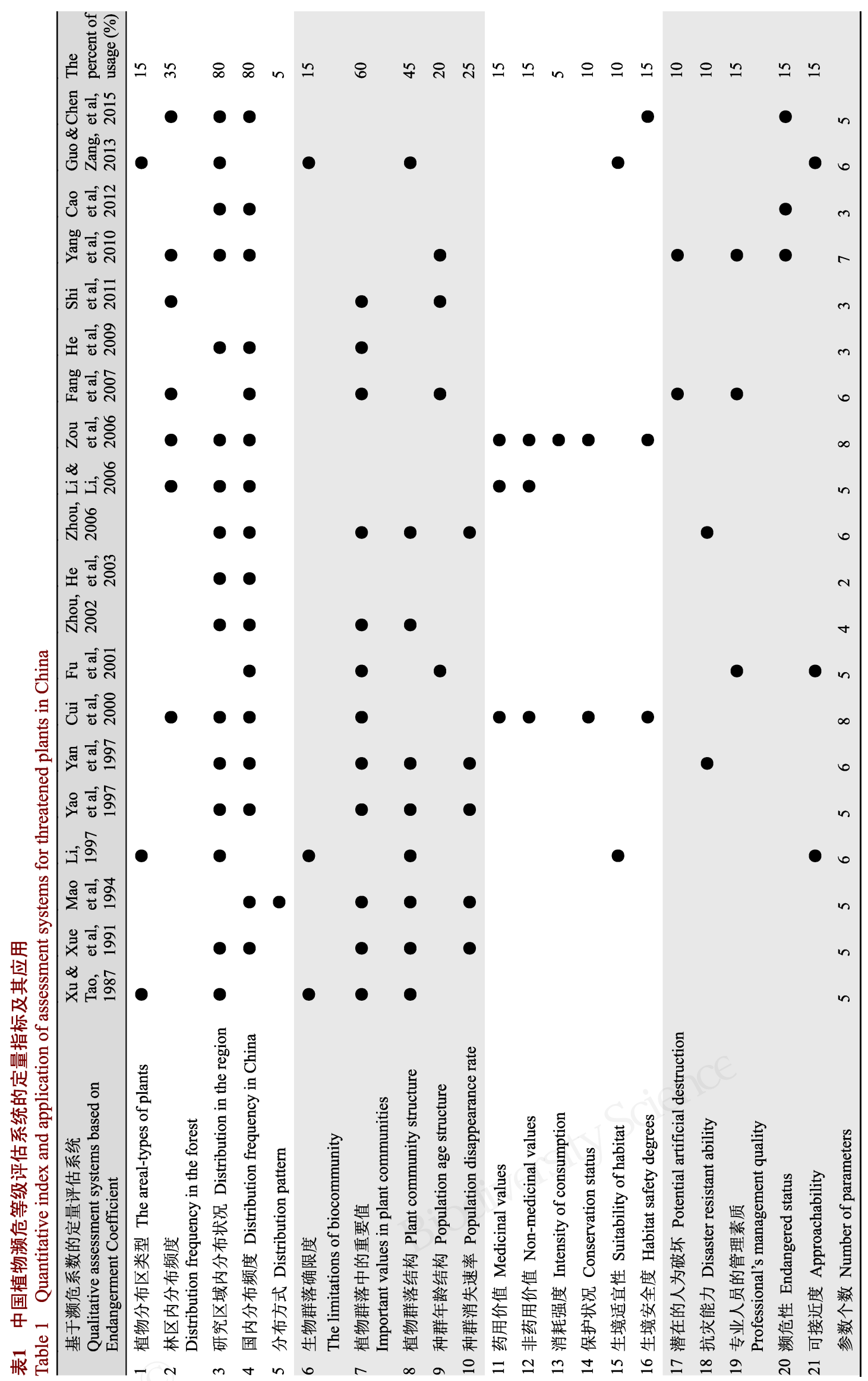




\section{3 二级模糊评价法}

二级模糊评价法是层次分析法的进一步使用。 二级模糊评价法为研究和处理具有双重不确定性 的事物提供了新的方法, 其核心是用参数间的模糊 关系替代数学假设基础上求出的解析关系。将该方 法用于植物受威胁等级评估是将保护生物学的理 论和模糊数学的手段相结合, 制定物种濒危度的二 级模糊综合评价的数学模型, 并较为客观、灵敏地 计算出物种的濒危度和优先保护系数, 得到基于物 种受威胁等级的优先保护顺序。

魏宏图和金念慈(1994)首次将二级模糊评价法 应用于我国受威胁植物——银缕梅(Parrotia subaequalis)的评估。该法以对小种群绝灭有影响的各种 因素和物种存活条件的随机性(包括统计随机性、环 境随机性、自然灾害和遗传随机性四个方面)为理论 依据, 同时以模糊数学的手段为计算工具, 对我国 银缕梅的受威胁程度及其保护顺序进行定量评估。 之后, 在其他类群的评估中, 我国专家在二级模糊 评价法的基础上, 结合分类学家提出的影响受威胁 物种保护顺序的评价指标进行修改, 制定了较为合 理的基于二级模糊评价法的物种优先保护顺序的 评价指标(米风贤, $2009^{\circledR}$; 郭晓莉等, 2010)。主要原 理和步骤如下:

第一步, 划分因素集U及其子集 $U_{k}$ 。在受威胁 植物等级评估过程中提出物种受威胁的主要影响 因子并制定标准。魏宏图和金念慈(1994)提出了包 括生殖生物学与生态遗传学、进化与系统学、生态 地理学、人为影响、稀有濒危类型及保护管理等在 内的6类因子:

$$
\begin{aligned}
& \mathrm{U}=\left\{\mathrm{U}_{1}, \mathrm{U}_{2}, \mathrm{U}_{3}, \mathrm{U}_{4}, \mathrm{U}_{5}, \mathrm{U}_{6}\right\} \\
& \mathrm{U}_{3}=\left\{\mathrm{U}_{31}, \mathrm{U}_{32}, \mathrm{U}_{33}, \mathrm{U}_{34}, \mathrm{U}_{35}\right\}
\end{aligned}
$$

其中, $U$ 集合中的 6 个子集 $U_{1} 、 U_{2} 、 U_{3} 、 U_{4} 、 U_{5} 、 U_{6}$ 互不相交; 每个因素子集含有与之相关的影响因子, 6 个子集共计 20 个影响因子, 如 $\mathrm{U}_{3}$ 代表生态地理学 $\left(\mathrm{U}_{3}\right)$, 其中的 $\mathrm{u}_{31}$ 代表特有度(endemicity), $\mathrm{u}_{32}$ 代表狭 域分布 (stenochory), $\mathrm{u}_{33}$ 代表星散分布 (scattered areal), $\mathrm{u}_{34}$ 代表子遗性(relic nature), $\mathrm{u}_{35}$ 代表生态脆弱 性(site brittleness)。

第二步, 初级评判。影响因子的强度分级。首 先确定 $U_{k}$ 中各因子起作用的大小, 确定权重集合 $A_{k}$,

(1) 米风贤 (2009) 河北珍稀濒危药用植物资源研究. 硕士学位论文, 河 北师范大学, 石家庄.
对 $\mathrm{U}_{\mathrm{k}}$ 中各个因素评定出评价集各个等级的隶属度, 构成单因素评判矩阵 $\mathrm{R}_{\mathrm{k}}$ 后进行初级评判, 获得初级 评判值 $B_{k}$ 。

$$
\begin{aligned}
& A_{k}=\left\{a_{k 1}, a_{k 2}, a_{k 3} \cdots a_{k n}\right\} \\
& A_{k} \times R_{k}=B_{k}=\left\{b_{k 1}, b_{k 2}, b_{k 3} \cdots b_{k n}\right\}
\end{aligned}
$$

其中, $n 、 k$ 分别为第 $k$ 个因素子集的 $n$ 项影响因素。

魏宏图和金念慈(1994)在进行重要性评估代码 确定时，主要依据每个指标对绝灭概率的作用不同。

第三步，二级模糊综合评判。根据二级指标的 评估代码建立相应系数矩阵 $\mathrm{A}=\left\{\mathrm{a}_{1}, \mathrm{a}_{2}, \mathrm{a}_{3}, \cdots, \mathrm{a}_{\mathrm{n}}\right\}$, 再将一级评判中, 各类因素子集 $\mathrm{U}_{\mathrm{k}}$ 的评判结果 $\mathrm{B}_{\mathrm{k}}$ 构 成二级评判矩阵 $\mathrm{R}$, 并进行二级评判式:

$$
\begin{aligned}
& \mathrm{R}=\left\{\mathrm{B}_{1}, \mathrm{~B}_{2}, \mathrm{~B}_{3} \cdots \mathrm{B}_{\mathrm{n}}\right\} \\
& \mathrm{A} \times \mathrm{R}=\mathrm{B}=\left\{\mathrm{b}_{1}, \mathrm{~b}_{2}, \mathrm{~b}_{3} \cdots \mathrm{b}_{\mathrm{n}}\right\}
\end{aligned}
$$

其中 $\mathrm{B}$ 为最终的评判结果。

二级模糊评价法因如下原因在我国有一定程 度的应用: 首先, 运用模糊数学理论将各因素判断 矩阵的权向量作为评价指标的权重, 将人的主观性 进行数学表达, 得出了各因素对评价指标影响程度 的顺序，使受威胁程度的等级评价更加客观、准确。 其次, 二级模糊评价法并不是纯粹的数学模型应用, 而是需要掌握包括物种分布、研究区域水平上物种 的受威胁程度、保护价值等多方面信息在内的物种 信息，这让评估结果更具科学意义。第三，在操作 上，二级模糊评价法采取了二层指标的综合评价， 不仅在第一层次指标间进行了权重分配，也根据指 标的重要程度对第二层次指标进行权重分配，且较 高层次的值与较低的评价结果息息相关。第四, 在 计算模糊值及系数设定时采取了矩阵计算, 这样让 同一类因子彼此关联更紧密，可以得出较为合理的 评判结果。最后, 规范化的操作还可以推动植物受 威胁及优先保护研究的规范化。当然, 二级模糊评 价法也存在一定的弊端，即对物种受威胁等级的定 量评估带有一定的片面性、局限性及模糊性(刘小雄 等, 2001), 特别是在指标选择方面存在较大的差异 和侧重。此外, 二级模糊评价法的数学原理较复杂, 理解上可能比较困难，所以并不具备良好的推广 条件。

\section{4 专家系统}

专家系统是人工智能(Artificial Intelligence, 简 称 $\mathrm{AI}$ )的一个分支, 是一类模仿专家解决问题的计 算机程序，其内部具有大量专业领域的知识，它能 
利用专家先验知识来解决该领域的实际问题(杨兴 等, 2007)。

毛夏等(1994)在前人的基础上将专家系统引入 我国植物受威胁等级评估的研究中, 探索出一套基 于专家系统的受威胁植物评定分级方法。该方法带 有初步智能的分级评估指标系统(计算机智能程序), 以评估指标体系为基础，加入人类专家的评价经验， 整体上模拟人类专家的思维过程。具体原理及过程 是: 初步选择基本指标, 并在基本指标基础上根据 相关程度形成组合指标, 并就其权重系数广泛征求 国内植物、生态等领域有关专家的意见，设置基本 指标和组合指标二者的权重系数。其中基本指标有: 国内分布频度 $\left(\mathrm{D}_{3}\right)$ 、种群结构 $\left(\mathrm{D}_{4}\right)$ 、现存多度 $\left(\mathrm{D}_{5}\right)$ 、 分布方式 $\left(\mathrm{D}_{6}\right)$ 、物种消失率 $\left(\mathrm{D}_{7}\right)$ 、种型情况 $\left(\mathrm{D}_{8}\right)$ 、特 有情况 $\left(\mathrm{D}_{9}\right)$ 、古老残遗情况 $\left(\mathrm{D}_{10}\right)$ 、利用价值 $\left(\mathrm{D}_{11}\right)$ 、 就地保护状况 $\left(\mathrm{D}_{12}\right)$ 、迁地保护状况 $\left(\mathrm{D}_{13}\right)$ 等; 组合指 标有: 濒危系数 $\left(\mathrm{D}_{16}\right)$ 、遗传损失系数 $\left(\mathrm{D}_{17}\right)$ 、价格系 数 $\left(\mathrm{D}_{18}\right)$ 、保护系数 $\left(\mathrm{D}_{19}\right)$ 四种。计算这两类指标的总 分指标。根据总分指标 $\left(\right.$ 含指标总分 $\mathrm{D}_{14}$ 和系数总分 $\mathrm{D}_{20}$ ) 初步划分受威胁物种的保护级别。分值特别高 的或特别低的物种，可以做到一次划分其保护级别, 但对于处于模糊区的物种, 如果不能清楚确定其等 级，则引入专家经验。针对处于模糊区间内的物种, 分级专家系统还设定了两个特殊的指标——最小 值数 $\mathrm{N}_{1}$ (基本指标中分值为 1 的个数)和最大值数 $\mathrm{N}_{2}$ (基本指标中分值达最高等级分的个数)。

专家系统的使用减轻了相关领域专家的工作 负担, 集中若干专家的经验组成知识处理系统, 以 多专家会诊的方式工作，系统具备的经验更为丰富， 解决问题的效率也更高。将专家系统与受威胁植物 等级评估的方法结合，相应地产生了受威胁物种评 估专家系统, 该系统兼具计算机的处理效率和受威 胁物种评估专家的知识及判断力。然而, 专家系统 也存在很大的缺陷, 特别是指标的选择与权重的确 定是通过专家咨询而得到的, 如果咨询面不够, 指 标选择不合理, 可能会对评估结果的影响比较大, 特别是处于模糊区间的物种, 往往会因为最小值数 或最大值数的个数而影响最终结果, 因此在我国受 威胁植物评估中的应用比较少。

\section{5 其他定量评估方法}

除了以上几种评估系统/方法外, 我国还有不 少成型的物种受威胁等级评估系统。这些物种受威
胁等级评估系统因原理比较复杂等原因, 并没有得 到推广使用, 如蒋志刚和栎恩源(2003)提出评估时 应区分生态濒危物种、进化濒危物种; 对于不同生 物类群，应区分物种的生活史对策，制定不同生活 史物种的濒危标准。在分析生境面积下降速率和种 群下降速率时，对上述两类物种分别根据不同的时 间尺度进行分析，制定不同生活史物种的受威胁标 准, 并区别对待特有物种。这些评估系统有些具有 较好的侧重点, 弥补了许多定量评估方法的不足, 为我国物种受威胁等级的评估提供重要思路, 但也 存在系统原理比较复杂或者部分物种信息难以获 得等问题，限制了系统/方法的推广应用。

\section{4 结语}

我国植物受威胁定量评估系统种类较多，但因 复杂度、科学性或是推广力度等原因, 并没有得到 普及。因此，到目前为止，我国并没有一套完整统 一的、等级标准固定的、得到大部分专家认可的植 物受威胁等级定量评估指标体系。

国内定量评估系统与国际上已经发展得较为 成熟的定量评估系统(如IUCN红色名录评估系统) 相比，还需进一步完善，特别是物种居群在时间尺 度上的变化。在使用IUCN红色名录评估系统的标 准 $\mathrm{A}$ 时，评估人员可根据已掌握的直接信息或者间 接信息估计(推测)物种在过去(标准A1和A2)和将来 (标准A3)可能的受威胁状况，甚至可结合过去将来 (A4)的变化趋势评估物种的受威胁状况，而本土定 量评估系统几乎没有反映物种居群动态变化、栖息 地动态变化的信息。同时，指标的选择不宜过多， 因为部分具有短板效应的指标可能会完全改变最 终的评估结果，因此建议选择几个重要的且能代表 物种野外居群现状的信息指标即可。当然, 国内评 估系统也有值得红色名录评估系统借鉴的地方, 如 国内评估系统常常使用的研究区域分布状态和分 布频度等指标(表1)是比较容易获取的信息，对于因 数据缺乏而被IUCN红色名录评估系统评估为DD等 级的物种来说，具有补充作用。

通过全面分析国内植物受威胁等级定量评估 系统与国外几种广泛应用的植物受威胁等级定量 评估系统可知，一个成熟的植物受威胁等级评估系 统必须具备以下要求: (1)受威胁等级的设置应该定 义明确、统一且合理，能够真实地反映整个物种受 
威胁状态，并且也方便地区之间的信息交流和合 作。此外, 物种受威胁等级应该与保护优先级进行 区分。国内的受威胁等级评估系统, 特别是基于濒 危系数的评估系统, 受威胁等级及保护优先级两个 概念有时候是混淆的。物种受威胁等级是物种保护 优先级必须考虑的一个重要的信息, 除此之外还要 考虑其他信息，如保护措施的有效性、经济价值、 社会认可度等(de Grammont \& Cuaron, 2006)。(2)评 估系统应该具备定量的评估标准, 标准应客观合理 且不冗余, 且应该与分布范围、个体数量等物种信 息相关。不应该列入模棱两可的信息，也就是不同 的评估者要能够通过同一套物种数据得到同一个 等级, 这样就能保证评估系统的准确性。(3)评估系 统能够在多个尺度范围内使用。在不同的地理尺度 上使用同一套评估标准时, 可能会出现在大的地理 尺度上受威胁而在小的地理尺度上不受威胁的结 果, 比如在IUCN红色名录评估系统中, 根据数量 和分布区指标存在全球性衰退的物种被划分到易 危, 在地区评估中可能因其族群数稳定而被定为无 危。反之, 也可能会有在大尺度地理范围不受威胁 而小尺度地理范围受威胁的结果。因此, 在等级表 达方面应该有所创新，比如，同时表达出不同尺度 范围的等级。如美国自然保育协会评估系统在描述 物种受威胁等级时同时展示两个地理范围的等级 (Faber-Langendoen et al, 2012)。这种综合等级显示 方式可以反映物种在多尺度的受威胁状况, 在制定 全球保护行动和国家保护策略时，可以综合考虑物 种在不同尺度的受威胁状况，使保护措施更加具体， 更具针对性。(4)评估指标应该包含一系列动态信息 (IUCN, 2001, 2010; de Grammont \& Cuarón, 2006)。评 估人员可以基于已知的信息评估物种在过去和将 来的动态变化。如IUCN红色名录评估系统中, 通过 对三个受威胁等级的绝灭率进行量化分析(标准E), 就可以基于已知信息定量分析居群随时间的变化。

物种受威胁等级评估是一个任重道远的工作, 它为制定保护优先行动迈出了关键的一步, 能够为 稀缺的保护相关的信息提供必要的补充。如目前有 多少受威胁的物种、物种的受威胁程度、物种受到 的威胁因素、有多少已知的物种已经绝灭、哪些类 群受威胁情况比较严重、哪些常见的有特殊价值的 类群即将绝灭等等。然而, 获得物种的受威胁等级 只是工作的起点, 后续工作需要及时跟上, 如制订
规范的物种评价程序及配套的规章制度, 建立包括 分类学、生态学、保护生物学等相关领域的专家库 (尤其是包括专科、专属领域的研究专家和地方一级 的专家、科研及保护人员信息库), 并及时分析出需 要继续加大投入的物种类群及保护空缺，同时还应 该增加评价过程的透明度并广泛吸收各方面的意 见和信息反馈，制订有关数据采集、关键指标阈值 确定、物种评估等方面的规范性文件, 鼓励专家、 地方保护机构或感兴趣者提出有关物种的受威胁 状态评价申请, 进一步促进物种相关数据信息的采 集及物种受威胁状态评价工作的开展。

\section{5 展望}

物种受威胁等级名录对于国家制定物种保护 优先级必不可少。只有在同一评估系统下评估物种 受威胁状态，才能得到更加合理可靠的受威胁等级, 有限的保护资源才能分配得更加合理、高效。近年 来，随着生物多样性问题的区域化及全球化，各国 更倾向于加强与周边地区的合作，实现生物多样性 保护的区域化、国际化(马克平, 2013)。在全球生物 多样性保护信息共享和可持续发展的要求下，使用 同一个评估系统评估物种受威胁等级逐渐得到各 国的认同，特别是以IUCN红色名录为代表的全球 层面、区域层面、国家层面的评估系统越来越得到 认可和推广。加强区域物种受威胁等级评估工作的 合作是全球生物多样性保护工作的重点。作为生物 多样性大国，国内的植物受威胁等级定量评估系统 应该依据上述的四个原则继续完善，形成与IUCN 红色名录评估系统一样规范性的评估文件，同时积 极推广应用, 将理论研究与具体的保护行动结合起 来。此外，还应该采用全球认可的评估标准获得物 种受威胁等级信息，将本国生物多样性保护工作纳 入全球范围内，让保护工作更加合理。

\section{参考文献}

An HJ, Liu JH, Zhang T (2005) Study on definition of endangering standard of exclusive plants in Eastern AlashanWestern Erdos. Journal of Arid Land Resources and Environment, 19, 194-200. (in Chinese with English abstract) [安慧君, 刘佳慧, 张蹈 (2005) 东阿拉善-西鄂尔多斯狭 域特有植物濒危分级标准与优先保护级的确定研究. 干 旱区资源与环境, 19, 194-200.]

Cao W, Li Y, Cong XX (2012) Quantitative evaluation of conservation priority of the endangered plants in Northeast 
China. Forest Research, 25, 190-194. (in Chinese with English abstract) [曹伟, 李岩, 丛欣欣 (2012) 中国东北濒危 植物优先保护的定量评价. 林业科学研究, 25, 190-194.]

Chen RB, Zhang GF, Liu J, Li S (2015) Endangerment grade and protection priority of rare plant species in Baohuashan National Forest Park, Jiangsu Province. Journal of Ecology and Rural Environment, 31, 174-179. (in Chinese with English abstract) [陈瑞冰, 张光富, 刘娟, 李硕 (2015) 江 苏宝华山国家森林公园珍稀植物的濒危等级及优先保护. 生态与农村环境学报, 31, 174-179.]

Cui GF, Cheng KW, Lu DZ, Wang JZ, Li JQ (2000) Evaluation on threatened situation and protection classes of vegetation in Beijing Labagoumen Reserve. Journal of Beijing Forestry University, 22(4), 8-13. (in Chinese with English abstract) [崔国发, 成克武, 路端正, 王建中, 李俊清 (2000) 北京 喇叭沟门自然保护区植物濒危程度和保护级别研究. 北 京林业大学学报, 22(4), 8-13.]

Cui GF, Xing SH, Zhao B (2007) Plants and Vegetation Protection in Beijing Mountains. China Forestry Publishing House, Beijing. (in Chinese) [崔国发, 刑韶华, 赵勃 (2007) 北京山地植物和植被保护. 中国林业出版社, 北京.]

de Grammont PC, Cuarón AD (2006) An evaluation of threatened species categorization systems used on the American continent. Conservation Biology, 20, 14-27.

Faber-Langendoen D, Nichols J, Master L, Snow K, Tomaino A, Bittman R, Hammerson G, Heidel B, Ramsay L, Young B (2012) NatureServe Conservation Status Assessments: Methodology for Assigning Ranks. NatureServe, Arlington, VA.

Fang YP, Liu SY, Wang ZX, Lei Y, Man JS (2007) Quantitative assessment of priority for conservation of the national protected plants in Qizimeishan Mountain Nature Preserve. Acta Botanica Boreali-Occidentalia Sinica, 27, 348-355. (in Chinese with English abstract) [方元平, 刘胜祥, 汪正 祥, 雷耘, 满金山 (2007) 七姊妹山自然保护区野生保护 植物优先保护定量研究. 西北植物学报, 27, 348-355.]

Fu LK (1992) The Red Data Book of China's Plants, Vol. 1: Rare and Endangered Species. Science Press, Beijing.

Fu ZJ, Ge YG, Zhang P (2001) A quantitative analysis on priority of conservation of the national protected plants in Taibai Mountain. Journal of Xinyang Teachers College (Natural Science Edition), 14, 422-424. (in Chinese with English abstract) [傅志军, 葛永刚, 张萍 (2001) 太白山 国家保护植物优先保护顺序的定量分析. 信阳师范学院 学报(自然科学版), 14, 422-424.]

Guo XL, Zhao JC, Peng XJ (2010) Rare and endangered medicinal plants in Hebei Province, China. Journal of Arid Land Resources and Environment, 24(4), 144-149. (in Chinese with English abstract) [郭晓莉, 赵建成, 彭献军 (2010) 河北珍稀濒危药用植物资源研究. 干旱区资源与 环境, 24(4), 144-149.]

Guo Z, Zang RG (2013) Evaluation index system of endangered levels of the wild plants with tiny population in China.
Scientia Silvae Sinicae, 49(6), 10-17. (in Chinese with English abstract) [国政, 藏润国 (2013) 中国极小种群野 生植物濒危程度评价指标体系. 林业科学, 49(6), 10-17.]

He F, Liu XL, Qing HY, He YP, Cai XH, Long TL, Ma QY (2009) A discussion on distributing laws and protected grades of protection plants in Western Sichuan Province. Journal of Sichuan Forestry Science and Technology, 30(6), 34-39. (in Chinese with English abstract) [何飞, 刘兴良, 青华永, 何亚平, 蔡小虎, 隆廷伦, 马钦彦 (2009) 川西 保护植物分布规律及保护等级划分探讨. 四川林业科技, 30(6), 34-39.]

He P, Xiao YA, Li XH (2003) Quantitative study of conservation priority of the rare and threatened plants in Jiangxi Province. Journal of Wuhan Botanical Research, 21, 423-428. (in Chinese with English abstract) [何平, 肖宜安, 李晓红 (2003) 江西珍稀濒危植物优先保护定量研究. 武汉植物学研究, 21, 423-428.]

Huang WC, Zhou XY, Ni ZY, Shao L (2015) An assessment of the extinction risk of Calanthe from China based on specimens and field observations. Biodiversity Science, 23, 493-498. (in Chinese with English abstract) [黄卫昌, 周翔 宇, 倪子轶, 邵丽 (2015) 基于标本和分布信息评估中国 虾脊兰属植物的濒危状况. 生物多样性, 23, 493-498.]

IUCN (2001) IUCN Red List Categories and Criteria, Version 3.1. IUCN, Gland, Switzerland and Cambridge, UK. https: //portals.iucn.org/library/sites/library/files/documents/RL-20 01-001.pdf/. (accessed on 2017-07-17)

IUCN Standards and Petitions Subcommittee (2010) Guidelines for using the IUCN Red List Categories and Criteria. Version 8.1. Prepared by the Standards and Petitions Subcommittee in March 2010. http://intranet.iucn.org/webfiles/doc/ SSC/RedList/RedListGuidelines.pdf/. (accessed on 2010-08-05)

IUCN/UNEP, Fitter R, Fitter M (1987) The Road to Extinction, IUCN, Gland, Switzerland and Cambridge, UK.

IUCN, UNEP, WWF (1980) World Conservation Strategy. IUCN, Gland.

Jiang ZG, Fan EY (2003) Exploring the endangered species criteria: Rethinking the IUCN Red List Criteria. Biodiversity Science, 11, 383-392. (in Chinese with English abstract) [蒋 志刚, 謑恩源 (2003) 关于物种濒危等级标准之探讨 —对 IUCN物种濒危等级的思考. 生物多样性, 11 , 383-392.]

Li JW, Li JQ (2006) Forest Biodiversity Protection Research. China Forestry Publishing House, Beijing. (in Chinese) [李 景文, 李俊清 (2006) 森林生物多样性保护研究. 中国林 业出版社, 北京.]

Li XK (1997) A assessment of rare and endangered plants for priority of conservation in Guangxi. Journal of Guangxi Academy of Sciences, 13(3), 9-16. (in Chinese with English abstract) [李先琨 (1997) 广西珍稀濒危植物优先保护评 价. 广西科学院学报, 13(3), 9-16.]

Liu XX, Yan LH, Liu XP (2001) Study on superior conservation grading index of the rare plants. Journal of Xiangtan 
Normal University (Natural Science Edition), 23(2), 42-46. (in Chinese with English abstract) [刘小雄, 颜立红, 刘享 平 (2001) 珍稀植物优先保护分级指标的研究. 湘潭师 范学院学报(自然科学版), 23(2), 42-46.]

Lucas G, Synge (1978) The IUCN Plant Red Data Book. IUCN, Switzerland.

Ma KP (2013) On Asia Biodiversity Conservation and Database Network. Biodiversity Science, 21, 515-516. (in Chinese) [马克平 (2013) 亚洲生物多样性保护与信息网 络: ABCDNet. 生物多样性, 21, 515-516.]

Mace GM, Lande R (1991) Assessing extinction threats: Toward a revaluation of IUCN threatened species categories. Conservation Biology, 5, 148-157.

Mao X, Jiang MK, Zheng LX (1994) The evaluation and gradation expert system for rare and endangered plants. Rural Eco-Environment, 10(3), 18-21. (in Chinese with English abstract) [毛夏, 蒋明康, 郑龙翔 (1994) 珍稀濒危植物评 价分级专家系统研究. 农村生态环境 , 10(3), 18-21.]

Miller RM, Rodríguez JP, Aniskowicz-Fowler T, Bambaradeniya C, Boles R, Eaton MA, Gärdenfors U, Keller V, Molur S, Walker S, Pollock C (2006) Extinction risk and conservation priorities. Science, 313, 439-442.

Miller RM, Rodríguez JP, Aniskowicz-Fowler T, Bambaradeniya C, Boles R, Eaton MA, Gärdenfors U, Keller V, Molur S, Walker S, Pollock C (2007) National threatened species listing base on IUCN criteria and regional guidelines: Current status and future perspectives. Conservation Biology, 21, 684-696.

Ministry of Environmental Protection of the People's Republic of China, Institute of Botany of the Chinese Academy of Sciences (1987) China List of Rare and Endangered Plants, Vol. 1. Science Press, Beijing. (in Chinese) [国家环境保护 局, 中国科学院植物研究所 (1987) 中国珍稀濒危保护 植物名录(第一册). 科学出版社, 北京.]

Onana JM, Cheek M (2011) Red List Book of the Flowering Plants of Cameroon: IUCN Global Assessments. Kew Publishing, London.

Peng XJ, Zhao JC, Li L, Sun YZ, Niu YL, Guo XL (2008) Assessment of Hebei flora against the IUCN Red List Criteria in China. Frontiers of Biology in China, 3, 176-186.

Perring FH, Farrell L (1983) British Red Data Books. 1. Vascular Plants, 2nd edn. Royal Society for Nature Conservation, Lincoln.

Qin HN, Zhao LN (2014) China higher plants and their threatened status. In: The Principles of Conservation Biology (eds Jiang ZG, Ma KP), pp. 117-148. Science Press, Beijing. (in Chinese) [覃海宁, 赵莉娜 (2014) 中国高等植物 及其濒危现状. 见: 保护生物学原理(蒋志刚, 马克平著), 117-148页. 科学出版社, 北京.]

Qin HN, Zhao LN (2017) Evaluating the threat status of higher plants in China. Biodiversity Science, 25, 689-695. (in Chinese with English abstract) [覃海宁, 赵莉娜 (2017) 中 国高等植物濒危状况评估. 生物多样性, 25, 689-695.]
Qin LW, Xiao Y, Fan YG, Zhao W, Zhao Y, Jin H, Yin H, Huang XT (2015) Assessment of the endangered status and conservation priorities for plants in the alpine tundra of Changbai Mountain. Acta Ecologica Sinica, 35, 134-141. (in Chinese with English abstract) [秦立武, 肖影, 范宇光, 赵伟, 赵荣, 金慧, 尹航, 黄祥童 (2015) 长白山高山苔 原带植物濒危现状及保护级别评估. 生态学报, 35, 134-141.]

Reveal JL (1981) The concepts of rarity and population threats in plant communities. In: Rare Plant Conservation: Geographical Organization (eds Morse LE, Henefin MS), pp. 41-47. The New York Botanical Garden, New York.

Rivers MC (2010) Subpopulations, locations and fragmentation: Applying IUCN Red List Criteria to herbarium specimen data. Biodiversity Conservation, 19, 2071-2085.

Shi DL, Mei H, Zhang Y, Deng CS, Lu YY, Meng FL, Hu L (2011) Quantitative study on priority for conservation of the wild protected plants in Houhe National Nature Reserve. Journal of Hubei Forestry Science and Technology, 168(2), 12-18. (in Chinese with English abstract) [石道良, 梅浩, 张云, 邓长胜, 路伊瑶, 孟凡琳, 胡凌 (2011) 后河自然 保护区野生保护植物优先保护定量研究. 湖北林业科技, 168(2), 12-18.]

State Forestry Bureau (2006) Standard on Classifying the Threatened Status of Wild Flora in China. Standards Press of China, Beijing. (in Chinese) [国家林业局 (2006) 中国 野生植物受威胁等级划分标准. 中国标准出版社, 北京.]

Tao L, Li XR, Liu XM, Ren J (2001) Quantitative study of conservation grading of rare and endangered desert plants in China. Scientia Silvae Sinicae, 37(1), 52-57. (in Chinese with English abstract) [陶玲, 李新荣, 刘新民, 任珺 (2001) 中国珍稀濒危荒漠植物保护等级的定量研究. 林 业科学, 37(1), 52-57.]

Tian GJ, Song YD, Liu Q, Zong XM, Zhang XJ, Meng XL, Geng DJ, Cao ZJ (2012) Investigation and evaluation of endangered diversity in Shandong Province. Ecology and Environmental Sciences, 21(1), 27-32. (in Chinese with English abstract) [田贵全, 宋沿东, 刘强, 宗雪梅, 张学 杰, 孟祥亮, 耿德江, 曹振杰 (2012) 山东省濒危物种多 样性调查与评价. 生态环境学报, 21(1), 27-32.]

Wang JR, Liu Y, Bau T (2015) Evaluation of endangered status and conservation priority of macro fungi in Shandong Province, China. Acta Ecologica Sinica, 35, 837-848. (in Chinese with English abstract) [王建瑞, 刘宇, 图力古尔 (2015) 山东省大型真菌物种濒危程度与优先保育评价. 生态学报, 35, 837-848.]

Wang S, Xie Y (2004) China Species Red List, Vol. 1: Red List. Higher Education Press, Beijing. (in Chinese) [汪松, 解炎 (2004) 中国物种红色名录, 第1卷: 红色名录. 高 等教育出版社, 北京.]

Wei HT, Jin NC (1994) Quantitative analysis of risk size of plant specie: A case of Shaniodendron subaequale M. B. Deng, H. T. Wei et X. Q. Wang. Journal of Plant Resources 
and Environment, 3(3), 1-8. (in Chinese with English abstract) [魏宏图, 金念慈 (1994) 银缕梅物种濒危度的 定量分析，植物资源与环境，3(3), 1-8.]

Wijnstekers W (2000) The evolution of CITES: A Reference to the Convention on International Trade in Endangered Species. CITES, Lausanne, Switzerland.

Xu ZF, Tao GD (1987) Discussion on the method of systematic assessment to regional threatened plants and their prior conservation. Acta Botanica Yunnanica, 9, 193-202. (in Chinese with English abstract) [许再富, 陶国达 (1987) 地 区性的植物受威胁及优先保护综合评价方法探讨. 云南 植物研究, 9, 193-202.]

Xue DY, Jiang MK, Li ZF (1991) Study on the grading indexes for the rare and endangered plants in Jiangsu, Zhejiang and Anhui provinces. China Environmental Science, 11(3), 161-166. (in Chinese with English abstract) [薛达元, 蒋明 康, 李正方 (1991) 苏浙皖地区珍稀濒危植物分级指标 研究. 中国环境科学, 11(3), 161-166.]

Yan LH, Peng CL, Xia XM, Liao FL (1997) Study on superior conservation grading index of the rare plants of Hunan. Hunan Forestry Science \& Technology, 24(3), 39-43. (in Chinese with English abstract) [颜立红, 彭春良, 夏晓敏, 廖舫林 (1997) 湖南珍稀濒危植物优先护存分级指标的 研究. 湖南林业科技, 24(3), 39-43.]

Yang H, Li PX, Dai HT (2010) Quantitative assessment for priority conservation of the wild protected plants in Jigong Mountain Nature Preserve. Central South Forest Inventory and Planning, 29(4), 50-54. (in Chinese with English abstract) [杨怀, 李培学, 戴慧堂 (2010) 河南鸡公山自然 保护区野生保护植物优先保护定量分析. 中南林业调查 规划, 29(4), 50-54.]

Yang X, Zhu DQ, Sang QB (2007) Research and prospect of expert system. Application Research of Computers, 24(5), 4-9. (in Chinese with English abstract) [杨兴, 朱大奇, 桑 庆兵 (2007) 专家系统研究现状与展望. 计算机应用研 究, 24(5), 4-9.]

Yao ZS, Zhang CQ, Ge F (1997) Priority assessment for the conservation of endangered medicinal plants in Jinggang Mountains, Jiangxi Province. Resources and Environment in Yangtze Valley, 6, 239-246. (in Chinese with English abstract) [姚振生, 张琮琼, 葛菲 (1997) 井冈山濒危药用 植物优先保护评价. 长江流域资源与环境, 6, 239-246.]
Zang CX, Cai L, Li JQ, Wu XP, Li XG, Li JS (2016) Preparation of the China Biodiversity Red List and its significance for biodiversity conservation within China. Biodiversity Science, 24, 610-614. (in Chinese with English

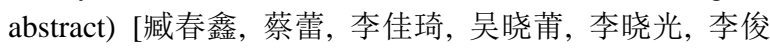
生 (2016) 《中国生物多样性红色名录》的制定及其对生 物多样性保护的意义. 生物多样性, 24, 610-614.]

Zhao LN, Li JY, Liu HY, Qin HN (2016) Distribution, congruence, and hotspots of higher plants in China. Scientific Reports, 6, 19080.

Zhong H, Zhou B (2010) Extinction risk assessment of Pinaceae Plants in National Key Protected Wild Plants List. Journal of West China Forestry Science, 39(3), 76-78. (in Chinese with English abstract) [钟华, 周涁 (2010) 国家重 点保护野生植物名录中松科植物濒危状态评估. 西部林 业科学, 39(3), 76-78.]

Zhou XR (2002) The research on the sequences of preferential protection of the rare and endangered plants in Sichuan. Life Science Research, 6, 94-97. (in Chinese with English abstract) [周先容 (2002) 四川省珍稀濒危植物优先保护 序列的研究. 生命科学研究, 6, 94-97.]

Zhou Y (2006) The research on the sequences of preferential protection of the rare and endangered plants in Changbai Mountains. Forest Research, 19, 740-749. (in Chinese with English abstract) [周猺 (2006) 长白山区珍稀濒危植物优 先保护序列的研究. 林业科学研究, 19, 740-749.]

Zhuang P, Wu H (1992) Priority assessment for conservation of threatened species at Emei Mountain. Resources Development and Conservation, 8(1), 53-56. (in Chinese with English abstract) [庄平, 吴荭 (1992) 峨眉山受威胁植物 优先保护评价. 资源开发与保护, 8(1), 53-56.]

Zou DL, He YJ, Lin QW, Cui GF, Zheng J, Dong JS, Li YB, He WC, Li CM, Zhang SX (2006) Evaluation on threatened situation and protection classes of the plants in Makehe Forest Region of Sanjiangyuan Nature Reserve, China. Journal of Beijing Forestry University, 28(3), 20-25. (in Chinese with English abstract) [邹大林, 何友均, 林秦文, 崔国发, 郑杰, 董建生, 李永波, 赫万成, 李长明, 张世 坌 (2006) 三江源玛可河林区植物濒危程度和保护类别 评价. 北京林业大学, 28(3), 20-25.]

(责任编委：蔵润国 责任编辑：黄祥忠) 